\title{
Increased level of serum prostaglandin-2 in early stage of esophageal squamous cell carcinoma
}

\author{
Dorota Diakowska, Krystyna Markocka-Mączka, Mirosław Nienartowicz, Andrzej Lewandowski, \\ Krzysztof Grabowski
}

Department of Gastrointestinal and General Surgery, Medical University of Wroclaw, Wroclaw, Poland

Submitted: 9 March 2012

Accepted: 26 September 2012

Arch Med Sci 2014; 10, 5: 956-961

DOI: 10.5114/aoms.2013.34985

Copyright @ 2014 Termedia \& Banach

\section{Abstract}

Introduction: Prostaglandin-2 (PGE-2), one of the products of cyclooxygenase2 (COX-2) induced catalysis, may play a critical role in the carcinogenesis of esophageal squamous cell carcinoma (ESCC). We investigated the efficacy of using serum PGE-2 concentration as a biomarker for this cancer type.

Material and methods: Prostaglandin-2 levels were analyzed in the serum of 65 ESCC patients and in 47 healthy individuals. The concentrations of cyclooxygenase-2 (COX-2) were measured in tumor tissues and normal tissues obtained from 31 surgically treated ESCC patients.

Results: Serum PGE-2 concentration was significantly higher in ESCC patients than in control patients $(p=0.004)$, especially in the early stages $(I+I I)$ of cancer $(p<0.0001)$. We observed significant inverse relationships between serum PGE-2 levels and: tumor stage, primary tumor progression, lymph and distant metastasis. The COX-2 concentration was significantly elevated in tumors as compared to normal tissues $(p=0.008)$. A significant correlation between serum PGE-2 and tumor COX-2 was observed (rho $=0.46, p=0.009$ ). However, ROC analysis showed that serum PGE-2 may be a weak prognostic factor for ESCC. Conclusions: Our results suggest that an elevated concentration of serum PGE-2 in the early stages of cancer may possibly be associated with tumor initiation and cancer development in ESCC. The exact role of these findings in early detection of this highly lethal cancer requires further research.

Key words: prostaglandin-2, cyclooxygenase-2, squamous cell carcinoma, esophagus.

\section{Introduction}

Esophageal cancer is a highly aggressive malignant disease worldwide with a 5 -year survival rate of $10 \%$ to $15 \%[1,2]$. There are two types of esophageal cancer: squamous cell carcinoma and adenocarcinoma. Squamous cell carcinoma is the predominant histological type of esophageal cancer in Poland [3]. The etiology of esophageal squamous cell carcinoma (ESCC) is multifactorial, but alcohol consumption and cigarette smoking are leading risk factors $[1,4]$. They contain potent esophageal carcinogens, which can initiate chronic esophageal inflammation with stimulation of T-lymphocytes, natural killer (NK) cells and production of several cytokines. It is possible that these carcinogens have a potential effect on cyclooxygenase-2 (COX-2) and the biosynthesis of its metabolites, such as prostaglandin-2 (PGE-2) [1, 4].

\author{
Corresponding author: \\ Dorota Diakowska MD, PhD \\ Department of Gastrointestinal \\ and General Surgery \\ Medical University of Wroclaw \\ 66 Marii Skłodowskiej-Curie St \\ 50-369 Wroclaw, Poland \\ Phone: +48 717842750 \\ E-mail: \\ dorota.diakowska@umed.wroc.pl
}


The prognosis for patients with esophageal squamous cell carcinoma (ESCC) treated with the standard approaches of surgery and radiotherapy is poor due to early systemic dissemination of the disease $[2,5]$. Evaluation of biological markers in this cancer and elucidation of their relationship to pathological parameters and prognosis is therefore justified [6].

Cyclooxygenase-2, the inducible form of the COX enzymes, is normally absent in most cells but it is also highly expressed in response to inflammatory cytokines, growth factors and mitogens $[7,8]$. Several studies suggest the stimulatory role of COX-2 in tumorigenesis and angiogenesis [9, 10]. Increased COX-2 expression has been documented in various cancers and preneoplastic lesions including head and neck cancer [11], lung cancer [12] and ESCC [13].

Cyclooxygenase-2 catalyzes the conversion of arachidonic acid to prostaglandins and thromboxane $[6,12]$. Prostaglandin-2 (PGE-2) plays a crucial role in carcinogenesis through the stimulation of tumor invasion, promotion of angiogenesis and resistance to apoptosis [14-16]. The growth-promoting action of PGE-2 was observed in cultured esophageal squamous cell carcinoma cells [1]. Nevertheless, the clinicopathologic role of PGE-2 in ESCC must still be clarified. Potential tumor markers, such as PGE-2, in serum can be used as supportive evidence for endoscopic observations and other biological markers in ESCC.

In the present study, the concentration of PGE-2 in the serum of patients with ESCC was examined. The relationships between serum PGE-2 level and clinicopathologic features were analyzed.

Furthermore, we compared the levels of COX-2 in tumor tissues and paired noncancerous mucosa in surgically treated ESCC patients. The correlation between tumor COX-2 level and serum PGE-2 concentration in ESCC was analyzed. The possible diagnostic potential of serum PGE-2 was measured.

\section{Material and methods}

Blood samples were taken before any treatment from 65 patients with ESCC who were treated in the Department of Gastrointestinal and General Surgery from 2004 to 2010. There were 53 men and 12 women (median age: 61 years, $95 \% \mathrm{Cl}$ : 53-65) in the study group. The control group consisted of 47 healthy blood donors (39 men, 8 women, median age: 56 years, $95 \% \mathrm{Cl}$ : 42-63), whose sera were provided by the Regional Center of Blood Donation and Therapeutics in Wroclaw, Poland.

Among 65 ESCC patients, 53 (81.5\%) had positive history of smoking: 19 patients were active smokers and 34 used to smoke in the past but they stopped smoking not later than 7 years ago. In the control group 16 patients (34.8\%) smoked and 31 were non-smokers.
The test patients suffered from only one type of cancer - squamous cell carcinoma of the esophagus, with no other cancers or inflammatory diseases.

The tumor-node-metastasis (TNM) classification system of the International Union Against Cancer (UICC) was used for clinical and pathological staging [17]. We examined 23 patients with clinical stage I + II, 8 with stage III and 34 with stage IV.

Thirty-one ESCC patients were treated surgically with a curative resection. The cancer lesion among the operated patients was located in the upper-middle part (25.2\%) or in the middle-lower part $(74.8 \%)$ of the esophagus. The remaining patients with advanced cancer $(n=34)$ were treated by palliative methods. Tumor specimens and corresponding normal tissues were obtained from 31 patients.

The study protocol was approved by the Medical Ethics Committee of Silesian Piasts University of Medicine, Wroclaw, Poland. All cancer patients signed an agreement to participate in this study.

Fresh samples of carcinomas from mucosa and submucosal regions and normal mucosa, situated approximately $10 \mathrm{~cm}$ from the tumor, were frozen and stored at $-80^{\circ} \mathrm{C}$ until extraction. The samples were thawed and weighed for homogenization, and $1 \mathrm{ml}$ of cold 0.1M TRIS-HCl (pH 7.4) with $1 \mathrm{mM}$ EDTA and $0.1 \%(\mathrm{v} / \mathrm{v})$ Triton $\mathrm{X}-100$ was added per every $50 \mathrm{mg}$ of tissue. The samples were homogenized for 2 min on ice in a Potter $\mathrm{S}$ device. The homogenates were centrifuged at $1850 \times g$ for $10 \mathrm{~min}$ at $4^{\circ} \mathrm{C}$. The clear supernatants were used for cytokine analysis.

The blood samples were collected from the peripheral vein into sterile tubes. All blood samples were clotted (15 min, RT) and centrifuged (10 min, $900 \mathrm{xg}$ ) in order to separate the serum. Collected sera were stored at $-45^{\circ} \mathrm{C}$ until examination.

Concentrations of PGE-2 in serum were measured by ELISA test (R\&D Systems, Abingdon, UK) according to the manufacturer's instructions. For PGE-2, the sensitivity of the assay was $30.9 \mathrm{pg} / \mathrm{ml}$, the calculated overall intra-assay coefficient of variation was $6.1 \%$, and the calculated overall interassay coefficient of variation was $9.9 \%$.

The COX-2 concentrations in tissue homogenates were determined by ELISA test (IBL, Hamburg, Germany). All assay procedures were carried out according to the manufacturer's instructions. For COX-2, the sensitivity was $0.25 \mathrm{ng} / \mathrm{ml}$, the calculated overall intra-assay coefficient of variation was $8.4 \%$, and the calculated overall inter-assay coefficient of variation was $6.2 \%$.

\section{Statistical analysis}

Data distribution was analyzed with the ShapiroWilk normality test. Data of PGE-2 and COX-2 were 


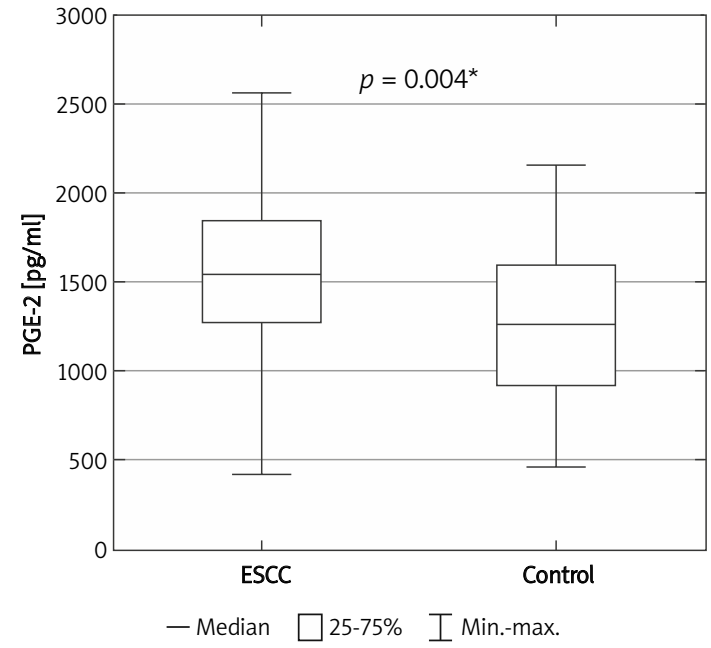

Figure 1. Serum PGE-2 concentration in ESCC patients and healthy controls

*Statistically significant

presented as median and $95 \%$ confidence interval $(95 \% \mathrm{Cl})$. Student's $t$-test for independent samples, Mann-Whitney $U$ test and ANOVA rank Kruskal-Wallis test were applied for comparisons of tested groups. Spearman's rank correlation test was used for correlation analysis.

Table I. Relationship between serum PGE-2 levels and clinicopathological parameters in ESCC patients

\begin{tabular}{|c|c|c|}
\hline \multirow[t]{2}{*}{ Parameter } & \multicolumn{2}{|c|}{ PGE-2 [pg/ml] } \\
\hline & Median $(95 \% \mathrm{Cl})$ & Value of $p$ \\
\hline \multicolumn{3}{|l|}{ Age: } \\
\hline$<60(n=33)$ & 1590 (1369-1817) & \multirow[t]{2}{*}{$0.004^{*}$} \\
\hline$\geq 60(n=32)$ & 1328 (1124-1523) & \\
\hline \multicolumn{3}{|l|}{ Gender: } \\
\hline Male $(n=53)$ & 1450 (1276-1593) & \multirow[t]{2}{*}{0.485} \\
\hline Female $(n=12)$ & 1560 (1106-2044) & \\
\hline \multicolumn{3}{|l|}{ Stage: } \\
\hline$I+\|(n=23)$ & 1870 (1640-2158) & \multirow[t]{3}{*}{$0.013^{*}$} \\
\hline III $(n=8)$ & 1234 (701-1661) & \\
\hline $\mathrm{IV}(n=34)$ & 1328 (1077-1383) & \\
\hline \multicolumn{3}{|c|}{ Tumor progression: } \\
\hline $\mathrm{T} 1+\mathrm{T} 2(n=18)$ & 1870 (1615-2259) & \multirow[t]{3}{*}{$0.004^{*}$} \\
\hline $\mathrm{T} 3(n=16)$ & $1495(1341-1736)$ & \\
\hline $\mathrm{T} 4(n=31)$ & 1265 (967-1319) & \\
\hline \multicolumn{3}{|c|}{ Lymph node metastasis: } \\
\hline NO $(n=22)$ & $1688(1456-2076)$ & \multirow[t]{2}{*}{$0.029^{\star}$} \\
\hline $\mathrm{N} 1(n=43)$ & 1335 (1154-1455) & \\
\hline \multicolumn{3}{|c|}{ Distant metastasis: } \\
\hline $\mathrm{MO}(n=35)$ & $1620(1451-1896)$ & \multirow[t]{2}{*}{$0.018^{*}$} \\
\hline $\mathrm{M} 1(n=30)$ & $1300(1046-1378)$ & \\
\hline
\end{tabular}

ANOVA rank Kruskal-Wallis test was employed, *statistically significant
The diagnostic utility for PGE-2 was designated by receiver operating characteristics (ROC) analysis and the area under the ROC curve (AUC) parameter. Additionally, cut-off values corresponding with the minimal false positive and false negative parameters were determined. The related sensitivity and specificity, and the likelihood ratios for positive $(L R+)$ and negative $(L R-)$ results were calculated.

Values of $p$ below 0.05 was considered as statistically significant. The statistical analyses were performed using Statistica 10 software (StatSoft Inc., Tulsa, USA).

\section{Results}

There were no significant differences in age and gender between ESCC patients and control subjects. The statistical analysis ( $t$-test for independent samples) of serum PGE-2 concentration did not demonstrate any significant difference between smokers and non-smokers among controls (1422 $\pm 456 \mathrm{pg} / \mathrm{ml}$ and $1187 \pm 448 \mathrm{pg} / \mathrm{ml}$ respectively, $p=0.096)$. Hence data from all persons included in the control group were used for the statistical analysis.

Serum PGE-2 concentration was significantly higher in patients with ESCC than in healthy control individuals (1540 pg/ml (1381-1662) vs. $1260 \mathrm{pg} / \mathrm{ml}$ (1132-1402), $p=0.004$ ) (Figure 1).

A comparison of PGE-2 levels in cancer patients at different stages of disease and the control group demonstrated that serum PGE-2 levels in patients at stages I + II were significantly higher than in healthy control individuals $(p<0.0001)$. In contrast, there were no significant differences in serum PGE-2 levels between ESCC patients at stage III or stage IV and the control group ( $p=0.609$ and $p=0.062$ respectively).

Table I shows the relationships between serum PGE-2 levels and clinical or pathological factors in ESCC patients. Serum PGE-2 levels significantly decreased with age, cancer stage, tumor progression, lymph node and distant metastasis. Patients at cancer stages I + II demonstrated significantly higher PGE-2 levels in comparison with stages III or IV ( $p=0.032$ and $p=0.002$, respectively). Serum PGE-2 concentration was also highest in patients with $\mathrm{T} 1+\mathrm{T} 2$ tumors as compared with $\mathrm{T} 3$ or $\mathrm{T} 4$ tumors ( $p=0.014$ and $p=0.002$, respectively).

Samples from esophageal tumors obtained after esophagectomy were processed along with tissues taken from corresponding normal mucosa for COX-2 concentration assays. The level of COX-2 significantly increased in tumor specimens in comparison to paired non-tumoral mucosa $(p=0.008)$ (Table II). A significant correlation (rho $=0.52$, $p=0.003$ ) between normal and tumor tissues was observed. There were no statistical differences in 
the relationships between COX-2 concentration and cancer stage, depth of tumor invasion or nodal status in tumor tissue.

Figure 2 demonstrates a significant association between serum PGE-2 and tumor COX-2 (rho $=0.46$, $p=0.009)$ in esophagectomy treated ESCC patients.

Table III presents the diagnostic potential of PGE-2 in ESCC patients. The area under the ROC curve (AUC) for PGE-2 is not high (0.725). The diagnostic sensitivity for PGE-2 is insufficient (only $32.3 \%$ ). The ROC analysis revealed that serum PGE-2 cannot be a favorable marker of ESCC presence.

\section{Discussion}

Many studies have reported that ESCC cancer cells are characterized by high proliferation capacity [18]. Nowadays, the role of non-tumoral cells in the tumor microenvironment is studied. Recent studies demonstrate that cytokines produced in the tumor microenvironment participate in the regulation of tumor cell proliferation [19]. In the neoplastic lesions of cancer these factors may have an anticancer capacity, but under certain conditions they change and play a supportive role in the growth, invasion and metastasis of cancer. Kubota et al. [20] reported that overexpression of COX-2 by tumor cells and non-tumor cells stimulates microenvironmental changes in ESCC.

It has been demonstrated that cigarette smoking stimulates chronic inflammation and induces COX-2 expression in ESCC [1, 4, 21]. Elevated expression of COX-2 in preneoplastic and neoplastic cells leads to $P G E-2$ production and activates tumor growth processes $[1,22]$. Our study revealed that the PGE-2 level is elevated significantly in the serum of ESCC patients in comparison to the control group. In our study $81.5 \%$ of ESCC patients smoked. This result confirms that an exogenous toxic factor, such as tobacco, can induce ESCC development.

Previous studies have reported that serum PGE-2 was elevated in squamous cell carcinoma of the head and neck [23, 24] and of the esophagus [25]. We first demonstrated that the serum PGE-2 level in ESCC was significantly higher in stages I + II, but not in stage III or IV in comparison with the control group. Our observations indicated that PGE-2 may exert a procarcinogenic effect during the early stages of this disease.

In the present study we found that the serum levels of PGE-2 in advanced ESCC (stages III and IV) were significantly lower than in stages I + II. Also serum levels of PGE-2 were negatively related to tumor progression, lymph node and distant metastasis in esophageal cancer. We suggest that the PGE-2 procarcinogenic effect may be revealed through the direct regulation of cellular proliferation and tumorigenesis.
Table II. Concentration of COX-2 in tumor tissue and paired non-cancerous mucosa

\begin{tabular}{|lc|}
\hline Tissue & COX-2 [ng/g tissue] \\
\hline Normal mucosa & $13.4(9.3-30.1)$ \\
\hline Tumor & $32.8(23.2-72.6)^{\star}$ \\
\hline
\end{tabular}

Data are presented as median (95\% CI); Mann-Whitney $U$ test was employed; ${ }^{*} p=0.008$ is considered as significantly different from normal mucosa

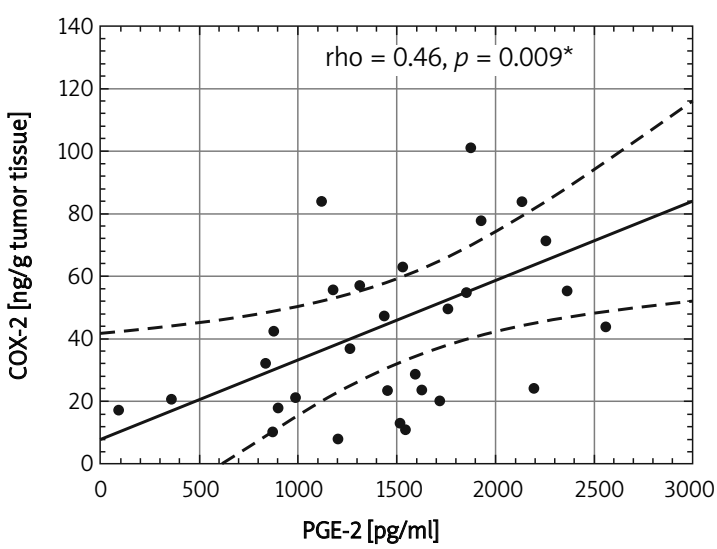

Figure 2. Correlation between tumor COX-2 and serum PGE-2 concentrations in operated ESCC patients

*Statistically significant

Table III. Diagnostic potential of PGE-2 as indicator of ESCC presence

\begin{tabular}{|lc|}
\hline AUC $(95 \% \mathrm{Cl})$ & $0.725(0.619-0.831)$ \\
\hline Value of $p$ for AUC $=0.5$ & $p<0.001$ \\
\hline Cut-off & $>1620$ \\
\hline Sensitivity (\%) & 32.3 \\
\hline Specificity (\%) & 96.2 \\
\hline LR+ & 8.40 \\
\hline LR- & 0.68 \\
\hline
\end{tabular}

$95 \% \mathrm{Cl}-95 \%$ confidence interval, AUC - area under ROC curve, $L R$ - likelihood ratios

To our knowledge, this is the first analysis of the relationship between serum PGE-2 and clinical and pathological parameters in ESCC patients. Studies by Hambek et al. on head and neck squamous cell carcinoma [23] demonstrated that serum PGE-2 levels significantly decreased from T1 to T4 tumor growth. They hypothesize that PGE-2 secretion was associated with tumor cells and the process of neovascularization during the early phases of tumor growth. Contradicting these results, Synderman [24] showed that tumor cells are not the major source of PGE-2. Rather, it is derived from tumor infiltrating and circulating inflammatory cells. Suppression of the host inflammatory response and less PGE-2 production is observed during the progression of cancer. 
Although PGE-2 may participate in "proliferative" carcinogenesis as a biochemical indicator, carcinogenesis is also stimulated by other factors. Highly proinflammatory cytokines are implicated in the pathogenesis of esophageal cancer, such as IL-6, IL-8, TGF- $\beta$ and others [1, 26-28]. Previously we have shown the proangiogenic potential and elevated expression of VEGF and IL-8 in patients with ESCC [27]. In this study we suggest that these factors are expressed not only in tumor cells but also in microenvironmental non-tumor cells. In early tumor initiation and development, the host immune system acts as a strong line of anticancer response. When cancer invasion and growth progresses, the host immune response changes and releases cytokines, chemokines, growth factors, etc., which stimulate cancer progression. It seems that PGE-2 is secreted mainly by tumor cells. In the early stages of cancer high PGE-2 levels may induce processes which inhibit the host immune system. When the host immune system changes, other factors may participate in cancer progression and PGE-2 levels can decrease. This may explain why the serum PGE-2 concentration is lower in advanced stages of cancer, while the remaining proinflammatory parameters increase.

We demonstrated that in operated patients with ESCC, COX-2 levels were higher in tumors than in corresponding normal mucosa. Our observations indicate that COX-2 overexpression is limited to cancer tissues. It has been previously reported that COX-2 expression increased in various malignances including head and neck cancer, lung cancer, gastric cancer, colorectal cancer and esophageal squamous cell carcinoma [10-13, 29].

Several studies have indicated that COX-2 overexpression is associated with a poor prognosis in early-stage carcinomas of esophageal cancer patients $[5,13]$. This suggests that COX-2 overexpression in earlier tumor stages can affect the disease progression or resistance to chemotherapy [13].

We analyzed COX-2 levels in tumor extracts in relation to various pathological features including depth of tumor invasion, lymph and distant metastasis and TNM stage. We found a lack of relationship between these parameters and COX-2 levels. Our data are in accordance with the immunohistological study of Shamma et al. [30]. They demonstrated a lack of significant correlations between COX-2 expression and selected clinicopathological parameters in squamous cell carcinoma of the esophagus. Moreover, they observed high expression of COX-2 in squamous dysplasia of esophagus and early esophageal cancer. However, the studies of Takatori et al. [31], who examined a large number of patients, showed that COX-2 overexpression was significantly correlated with depth of tumor invasion, tumor stage and survival in ESCC.
Our results demonstrated a significant correlation between tumor COX-2 concentration and corresponding normal mucosa. This fact suggests that each person may be susceptible to inducing COX-2 production. We used available tumor tissue samples and blood samples from operated patients to evaluate PGE-2 as an ESCC marker. We found a strong correlation between PGE-2 concentrations in serum and COX-2 levels in squamous cell cancer tissue. This result made the use of serum PGE-2 level as a potential marker of ESCC presence hopeful. However, ROC analysis showed that the use of serum PGE-2 concentration as a biomarker in ESCC is doubtful. The diagnostic parameters for PGE-2 were significantly lower than for other tested factors [26, 32, 33]. The best diagnostic potential for ESCC was found for C-reactive protein [28]. Our results suggest that $P G E-2$ cannot be an independent diagnostic factor for the presence of this type of cancer.

In conclusion, our findings suggest that the concentrations of serum PGE-2 increased significantly in the early stages of esophageal cancer. However, this factor cannot be used as a diagnostic parameter of ESCC presence. The exact role of these findings in early detection of this highly lethal cancer requires further research.

\section{References}

1. Yu L, Wu WK, Li ZJ, et al. E series of prostaglandin receptor 2-mediated activation of extracellular signal-regulated kinase/activator protein-1 signaling is required for the mitogenic action of prostaglandin E2 in esophageal squamous-cell carcinoma. Pharmacol Exp Ther 2008; 327: 258-67.

2. Bird-Lieberman EL, Fitzgerald RC. Early diagnosis of oesophageal cancer. Br J Cancer 2009; 101: 1-6.

3. Szumiło J. Epidemiology and risk factors of the esophageal squamous cell carcinoma. Pol Merkur Lekarski 2009; 26: 82-5.

4. Huang RY, Chen GG. Cigarette smoking, cyclooxygenase-2 pathway and cancer. Biochim Biophys Acta 2011; 1815: 158-69.

5. Huang JX, Xiao W, Chen WC, et al. Relationship between COX-2 and cell cycle-regulatory proteins in patients with esophageal squamous cell carcinoma. World J Gastroenterol 2010; 16: 5975-81.

6. Sun ZG, Song GM, Zhang M, Wang Z, Ni ZH. Clinical study on magnesium, copper and chrome levels in patients with oesophageal squamous cell carcinoma. Wspolczesna Onkol 2011; 15: 257-60.

7. Wang D, Mann JR, Dubois RN. The role of prostaglandins and other eicosanoids in the gastrointestinal tract. Gastroenterology 2005; 128: 1445-61.

8. Krawczyk-Rusiecka K, Lewiński A. Cyclooxygenase-2 expression and its association with thyroid lesions. Arch Med Sci 2010; 6: 653-7.

9. Patrignani P, Tacconelli S, Sciulli MG, Capone ML. New insights into COX-2 biology and inhibition. Brain Res Rev 2005; 48: 352-9. 
10. Cianchi F, Cuzzocrea S, Vinci MC, et al. Heterogeneous expression of cyclooxygenase-2 and inducible nitric oxide synthase within colorectal tumors: correlation with tumor angiogenesis. Dig Liver Dis 2010; 42: 20-7.

11. Abrahao AC, Castilho RM, Squarize $\mathrm{CH}$, Molinolo AA, Santos-Pinto D Jr, Gutkind JS. A role for COX2-derived PGE2 and PGE2-receptor subtypes in head and neck squamous carcinoma cell proliferation. Oral Oncol 2010; 46: 880-7.

12. Koch A, Gustafsson B, Fohlin H, Sörenson S. Cyclooxygenase-2 expression in lung cancer cells evaluated by immunocytochemistry. Diagn Cytopathol 2011; 39: 188-93.

13. Kuo KT, Chow KC, Wu YC, et al. Clinicopathologic significance of cyclooxygenase-2 overexpression in esophageal squamous cell carcinoma. Ann Thorac Surg 2003; 76: 909-14.

14. Kuo KT, Wang HW, Chou TY, et al. Prognostic role of PGE2 receptor EP2 in esophageal squamous cell carcinoma. Ann Surg Oncol 2009; 16: 352-60.

15. Wu BW, Li DF, Ke ZF, et al. Expression characteristics of heparanase in colon carcinoma and its close relationship with cyclooxygenase- 2 and angiogenesis. Hepatogastroenterology 2010; 57: 1510-4.

16. Brecht K, Weigert A, Hu J, et al. Macrophages programmed by apoptotic cells promote angiogenesis via prostaglandin E2. FASEB J 2011; 25: 2408-17.

17. Sobin LH, Wittekind CH. TNM classification of malignant tumors. $6^{\text {th }}$ edn. Hoeboken, New Jersey, Jon Wiley\&Sons 2002.

18. Noma K, Smalley KS, Lioni M, et al. The essential role of fibroblasts in esophageal squamous cell carcinoma-induced angiogenesis. Gastroenterology 2008; 134: 1981-93.

19. Liu J, Li Z, Cui J, Xu G, Cui G. Cellular changes in the tumor microenvironment of human esophageal squamous cell carcinomas. Tumor Biol 2012; 33: 495-505.

20. Kubota Y, Kaneko K, Konishi K, et al. The onset of angiogenesis in a multistep process of esophageal squamous cell carcinoma. Front Biosci 2009; 14: 3872-8.

21. Lambert R. Epidemiology of oesophagogastric cancer. Best Practice Res Clin Gastroenterol 2007; 21 921-45.

22. Müller-Decker K. Cyclooxygenase-dependent signaling is causally linked to non-melanoma skin carcinogenesis: pharmacological, genetic, and clinical evidence. Cancer Metastasis Rev 2011; 30: 343-61.

23. Hambek M, Baghi M, Wagenblast J, Schmitt J, Baumann H, Knecht R. Inverse correlation between serum PGE2 and T classification in head and neck cancer. Head Neck 2007; 29: 244-8.

24. Snyderman C. Serum PGE2 levels are inversely correlated with $T$ classification in patients with squamous cell carcinoma of the head and neck. Head Neck 2008; 30: 132-3.

25. Morgan G. Deleterious effects of prostaglandin E2 in oesophageal carcinogenesis. Med Hypoth 1997; 48: 177-81.

26. Krzystek-Korpacka M, Matusiewicz M, Diakowska D, et al. Elevation of circulating interleukin-8 is related to lymph node and distant metastases in esophageal squamous cell carcinomas: implication for clinical evaluation of cancer patient. Cytokine 2008; 41: 232-9.

27. Diakowska D, Lewandowski A, Markocka-Mączka K, Grabowski K. Elevated levels of serum transforming growth factor-beta 1 in oesophageal squamous cell carcinoma patients. Wspolczesna Onkol 2011; 15: 137-41.

28. Fujiwara $\mathrm{H}$, Suchi K, Okamura S, et al. Elevated serum CRP levels after induction chemoradiotherapy reflect poor treatment response in association with IL-6 in serum and local tumor site in patients with advanced esophageal cancer. J Surg Oncol 2011; 103: 62-8.
29. Mrena J, Wiksten JP, Kokkola A, Nordling S, Ristimäki A, Haglund C. COX-2 is associated with proliferation and apoptosis markers and serves as an independent prognostic factor in gastric cancer. Tumor Biol 2010; 31: 1-7.

30. Shamma A, Yamamoto H, Doki Y, et al. Up-regulation of cyclooxygenase- 2 in squamous carcinogenesis of the esophagus. Clin Cancer Res 2000; 6: 1229-38.

31. Takatori $\mathrm{H}$, Natsugoe S, Okumura $\mathrm{H}$, et al. Cyclooxygenase-2 expression is related to prognosis in patients with esophageal squamous cell carcinoma. Eur J Surg Oncol 2008; 34: 397-402.

32. Kupisz K, Stepulak A, Zdunek M, Klatka J. Preliminary results of prognostic significance of proliferating cell nuclear antigen expression in advanced primary larynx carcinomas and lymph node metastases. Arch Med Sci 2010; 6: 65-70.

33. Mroczko B, Kozłowski M, Groblewska M, et al. The diagnostic value of the measurement of matrix metalloproteinase 9 (MMP-9), squamous cell cancer antigen (SCC) and carcinoembryonic antigen (CEA) in the sera of esophageal cancer patients. Clin Chim Acta 2008; 389: 61-6. 